\title{
Ambulatory Blood Pressure Monitoring
}

\author{
Denise Janicki-DeVerts
}

ThOMAS W. Kamarck

A ccumulating evidence on the prognostic utility of ambulatory blood pressure (ABP) is beginning to shift the consensus on its potential value for clinical practice. This trend is evidenced by the increasing use of $\mathrm{ABP}$ monitoring (ABPM) (Mancia, Di Rienzo, \& Parati, 1993) and by new Medicare policies approving reimbursement of ABPM (CMS, 2001; Pickering et al., 2005). Concurrently, new technologies and methodologies are providing us with the means of examining the determinants of ABP with greater precision (Fahrenberg \& Myrtek, 2001; Shiffman \& Stone, 1998). These converging trends yield important opportunities for behavioral medicine research. The purpose of this chapter is to explore the role of ABPM in contemporary behavioral medicine research and to outline a number of methodological considerations that are critical for designing and implementing studies in this area.

\section{WHY STUDY AMBULATORY BP?}

Since 1966, when the results of automated ABP measures first were compared with clinic BP assessments (Sokolow, Werdegar, Kain, \& Hinman, 1966), numerous studies have shown that mean 24-hour ABP is a stronger correlate of end organ damage (such as left ventricular hypertrophy) than is BP measured in the clinic (Mancia \& Parati, 2000). The prognostic utility of ABPM has been demonstrated as well. For example, a number of studies conducted with hypertensive samples have shown that 24-hour ABP measures are more strongly associated with cardiovascular morbidity and mortality than

AUTHOR'S NOTE: This work was supported by the National Heart, Lung, and Blood Institute research grants HL56346 and HL040962, by an NHLBI training fellowship (HL07560), and by the Pittsburgh Mind-Body Center (HL076852 [University of Pittsburgh], HL076858 [Carnegie Mellon University). 
clinic-based measures and/or they are independent predictors of cardiovascular health outcomes in hypertensive persons after controlling for clinic pressures (Clement et al., 2003; Khattar, Senior, \& Lahiri, 1998; Perloff, Sokolow, \& Cowan, 1991; Redon et al., 1998; Staessen et al., 1999; Verdecchia, Porcellati, Schillaci et al., 1994; Verdecchia et al., 2002). A small but growing body of evidence has recently developed demonstrating the prognostic value of ABP in the general population as well (Hansen, Jeppesen, Rasmussen, Ibsen, \& Torp-Pedersen, 2005; Ohkubo et al., 2000; Sega et al., 2005). While most of these studies have used 24hour averages for their assessment of ABP, some have also examined daytime and nighttime BP separately. Findings are inconsistent regarding the relative importance of daytime and nighttime ABP, with some (Sega et al., 2005; Staessen et al., 1999) showing that nighttime $\mathrm{BP}$ is more predictive than daytime ABP and others (Fagard, Bielen, \& Amery, 1992; Hansen et al., 2005) showing that ABPs during the two periods of the day are equivalent in terms of their prognostic value.

That ABP readings may be more strongly predictive of future cardiovascular morbidity and mortality raises questions about the comparability of ambulatory and clinic assessments. Indeed, comparisons of the values obtained across these two methods of BP measurement reveal systematic differences. Because clinic BPs tend to be higher than pressures taken during daily life (a phenomenon dubbed the white coat effect), the American Society of Hypertension recommends that lower values be used to define the upper limits of normal for daytime ABP $(<135 / 85)$ compared with clinic pressures (<140/80) (Pickering, 1996).

More important, the literature on the prognostic utility of ABP also raises questions about the value of relying on clinic measures alone in diagnosing hypertension and identifying those in need of treatment. White coat bypertension (WCH), a variant of the white coat effect, is said to occur in individuals for whom clinic BP values are in the hypertensive range while ABP values are normal. Fifteen to $20 \%$ of persons diagnosed with Stage 1 hypertension (140-159 $\mathrm{mmHg}$ systolic BP [SBP] or $90-99 \mathrm{mmHg}$ diastolic BP [DBP]; Joint National Committee [JNC], 1997) have been shown to fall within this group (Pickering et al., 2005), and the prevalence may be as high as $60 \%$ to $80 \%$ among persons with borderline increases in clinic BP (Staessen, O'Brien, Thijs, \& Fagard, 2000). Identification of WCH is important insofar as persons with $\mathrm{WCH}$ have been shown to be at reduced risk for cardiovascular events compared to individuals with more consistently elevated pressure. In the Syst-Eur Trial, for example, the risk associated with clinic SBP was reduced by $20 \%$ for each $10-\mathrm{mmHg}$ discrepancy between daytime ambulatory and clinic BP (Staessen et al., 1999). It is not clear whether the risk associated with $\mathrm{WCH}$ is comparable to that of individuals with normal clinic pressures, or whether the $\mathrm{WCH}$ group is at an intermediate level of risk (Pickering et al., 2005).

A smaller but equally important phenomenon is represented by persons showing normal clinic pressures but elevated pressures during ambulatory recording (masked hypertension). Emerging evidence suggests that this group also may be at elevated risk for clinically manifest cardiovascular disease (CVD) relative to those with $\mathrm{ABP}$ in the normal range, again reflecting the prognostic value of assessing $\mathrm{BP}$ during daily life (Bobrie et al., 2004).

ABP monitoring has permitted investigators to identify individuals whose BP profiles are characterized by unusual patterns of nocturnal as well as diurnal activity. BP typically declines as individuals transition from wakefulness to sleep, a phenomenon referred to as dipping (O’Brien, Sheridan, \& O'Malley, 1988). However, some individuals show an absence of nocturnal BP decline (nondipping, usually defined as a failure to show nighttime ABP decreases of $10 \%$ or more). Elevated 
nocturnal BP has been hypothesized to contribute to overall pressor load, hastening the development of target organ damage. For example, nondippers are overrepresented among those with damage to the kidney (microalbuminuria; Bianchi, Bigazzi, Baldari, Sgherri, \& Campese, 1994), heart (left ventricular hypertrophy; Verdecchia, Porcellati, Zampi, et al., 1994), and brain (silent lacunar infarction; Kario, Pickering et al., 2001; Pickering \& Kario, 2001). Nondipping is prospectively associated with increased risk for cardiovascular morbidity and mortality (Amar et al., 2000; Kario, Schwartz, Davidson, \& Pickering, 2001; Sturrock et al., 2000; Verdecchia, Porcellati, Schillaci et al., 1994), even among unselected community samples (Ohkubo et al., 1997).

According to recent consensus reports, identification of $\mathrm{WCH}$, masked hypertension, and nondipping BP pattern are among the most important clinically useful applications of ABPM (O'Brien et al., 2003; Pickering et al., 2005). JNC guidelines cite hypotensive symptoms following treatment, episodic hypertension, and autonomic dysfunction as additional indicators for use of ABPM (JNC, 1997).

\section{EXPLANATIONS FOR DIFFERENTIAL UTILITY OF ABP}

It frequently is assumed that the differential utility of $\mathrm{ABP}$ as a marker of CVD risk stems from the fact that ABP captures the effects on BP of lifestyle and behavioral influences that cannot be observed in the clinic (Devereux et al., 1983). Under the assumption that medical morbidity risk from hypertension increases as a function of cumulative BP exposure or "load," measurement conditions that will best reflect the degree of cumulative exposure should yield more accurate assessment of risk.

In addition to $\mathrm{ABP}$ measures being more representative of cumulative pressure exposure, other measurement features associated with ABPM also could account for its differential utility. Ambulatory measures typically are based on the average of 50 to 100 observations taken over the course of a day (Pickering et al., 2005), whereas clinic measures within a single session may be based on as few as one to three BP assessments collected over the course of several minutes. Because ABP readings are automated, measurements also may be more standardized and less prone to reader bias compared with clinic assessments (Fagard et al., 1992). Measurement standardization and use of multiple observations are both associated with enhanced reliability; therefore, each of these features of ABPM could plausibly contribute to the enhanced prognostic value of ABP.

Insofar as the methodological factors described here might account for the prognostic superiority of ABP, it is possible that similar prognostic effects might be obtained more efficiently simply by increasing the number of assessments in the clinic. A small number of studies have examined this possibility by augmenting the number of clinic BP readings taken within a given session or increasing the number of days of clinic BP assessment (Fagard et al., 1992; Fagard, Staessen, Thijs, \& Amery, 1995; Fagard, Staessen, \& Thijs, 1997). As expected, most of these studies showed some increase in the predictive power of clinic measures relative to ABP, when these methodological refinements were adopted (Fagard et al., 1992, 1997).

In an effort not only to minimize but to eliminate methodological differences between the two types of assessments, we recently compared the predictive power of ABP and clinic assessment when both methods employed the same types of measurement devices, the same number of observations, and the same intervals of measurement. Specifically, we sampled four automated readings over a 2.5 -hour period in the 
laboratory (clinic) and in the field (ABP) in a sample of 216 healthy older adults (ages 50-70) (Kamarck, Polk, Sutton-Tyrrell, \& Muldoon, 2002) using ambulatory BP devices within each setting. We averaged across the four laboratory readings to obtain measures of clinic BPs (SBP and DBP) for each person, and across the four field assessments, in turn, to obtain measures of ABP. Having controlled for methodological differences between these two types of assessment, we examined whether $\mathrm{ABP}$ continued to be superior to clinic BP in terms of its cross-sectional association with carotid artery intima medial thickness (IMT), as measured by ultrasonography. ${ }^{1}$

Even under the conservative conditions described here, the statistical association between BP and IMT was stronger when BP was measured in the field relative to in the clinic $(r=.30$ for SBP for ambulatory vs. $r=.23$ for clinic assessments). Moreover, ambulatory measures were stronger independent correlates of IMT than clinic measures in this study (ABP measures were significant correlates of IMT in multivariate models, which included clinic BP assessed by manual manometry, whereas the automated clinic measures were not significant predictors in the same models). Those in the top quartile of ambulatory SBP showed a significantly increased risk for observable carotid artery plaque as well as IMT, with the odds of observing one or more plaques being 4.7 times greater in the top quartile than in those in the bottom quarter of the distribution, even after adjusting for comparable clinic measures.

\section{WHY STUDY PSYCHOSOCIAL PROCESSES AND ABP?}

These findings, which rule out simple methodological explanations for the incremental effects of ABP, highlight instead the potential importance of daily life influences and, by extension, psychosocial processes, in accounting for the incremental prognostic value of ABP. The central role of ABP both as a prognostic marker and as a window on psychosocial processes that may contribute to cardiovascular risk marks this area of study as an important one for behavioral scientists interested in cardiovascular disease.

Our own work suggests that ABP may fluctuate with changes in psychosocial challenges during daily life, and that such associations may help explain some of the links between psychosocial variables and CVD risk (Kamarck et al., 2004, 2005). We and others have also examined psychosocial factors as predictors of ABP dipping (Ituarte, Kamarck, Thompson, \& Bacanu, 1999; Kario, Schwartz et al., 2001) under the assumption that nocturnal physiology may be altered by daytime behaviors and concerns (Hall et al., 2004). Overall, however, surprisingly little research, to date, has examined the extent to which daytime ABP or nocturnal changes in ABP may mediate the effects of psychosocial processes on CVD risk. These are questions that we believe have important clinical significance and thus are ripe for further investigation.

\section{METHODOLOGICAL CONSIDERATIONS}

In the following pages, we discuss several issues that are important considerations when planning and conducting an ABPM study. First, we discuss factors to consider when choosing an ambulatory monitor. Second, we describe the types of training that should be provided to research staff and study participants. Third, we discuss the importance of detecting artifactual BP readings, and list several methods that have been devised to handle them. Finally, we discuss a number of general design issues, as well as relevant data analytic strategies. 


\section{Choosing an Ambulatory Monitor}

Choice of an appropriate monitor is of paramount importance to ABPM. A number of factors influence this decision: (1) accuracy of monitor, (2) auscultatory or oscillometric detection techniques, (3) participant comfort, and (4) cost.

Accuracy of Monitor. The British Hypertension Society (BHS) protocol and the standard set by the United States Association for the Advancement of Medical Instrumentation (AAMI) are the two most widely used protocols for validating the accuracy of automated BP devices (AAMI, 1993; O'Brien et al., 1993). Briefly, both protocols involve assessing the agreement of automated devices with a mercury standard. For a device to be recommended, it must fulfill the AAMI criteria for both SBP and DBP, and receive a grade of $\mathrm{A}$ or $\mathrm{B}$ according to the BHS criteria for both parameters (O'Brien, Waeber, Parati, Staessen, \& Myers, 2001). In order to receive an A grade, $60 \%$ of all readings taken with the automated device must be within $\pm 5 \mathrm{mmHg}$ of the standard, $85 \%$ within $\pm 10 \mathrm{mmHg}$ of the standard, and $95 \%$ within $\pm 15 \mathrm{mmHg}$ of the standard. Corresponding percentages for a grade of $\mathrm{B}$ are $50 \%, 75 \%$, and $90 \%$, respectively (O'Brien et al., 2001). A list of currently available ambulatory monitors that have met BHS and AAMI criteria is available on the $d a b l^{\circledR}$ Educational Trust website (dabl Educational Trust).

Auscultatory Versus Oscillometric Techniques. Another factor to consider when choosing an ambulatory monitor is whether the device uses auscultatory or oscillometric detection methods. Auscultatory monitors detect the onset and disappearance of Korotkoff sounds (K-sounds) with a transducer (microphone) that is placed on the skin over the brachial artery and beneath the compression cuff. Thus, this automated technique mimics the standard auscultatory mercury column method that involves an observer detecting $\mathrm{K}$-sounds with a stethoscope. An advantage of auscultatory monitors that is relevant to ABPM is the relative resistance of auscultatory measurements to movement artifact during physical activity (Pickering et al., 2005). Microphone transducers are, however, sensitive to excess background noise or vibrations. Oscillometric monitors rely on oscillations in cuff pressure to detect SBP and DBP. Specifically, oscillations in cuff pressure during gradual deflation correspond to mean intraarterial pressure, such that oscillations begin at approximately SBP and continue below DBP. From the oscillations, SBP and DBP are estimated indirectly according to a manufacturer-specific, empirically derived algorithm. Oscillometric measurements are more likely to be influenced by arm movement than those taken with auscultatory devices. As readings do not require the use of a microphone, however, oscillometric measurements are not influenced by background noise.

Both auscultatory and oscillometric techniques have been validated insofar as readings from monitors using either detection method have been found to correlate reasonably well with intraarterial BP (Henschel, de la Vega, \& Taylor, 1954). However, auscultation has been found to be more accurate than oscillometry when validated against a standard mercury column (Imai et al., 1989; Keavney, Bird, Caiazza, Casadei, \& Conway, 2000; Pannarale et al., 1993). It is important to note that individual readings from either type of monitor may differ considerably from intraarterial standards.

Despite the generally superior accuracy of auscultatory monitors, most currently available ambulatory devices employ oscillometry. The shift toward oscillometry likely is due to the relative ease of cuff application: participants need not be trained in the proper positioning of an auscultatory transducer over the brachial artery. In addition to presenting less 
burden to the participant, this feature of oscillometric monitors may enhance data collection. Two studies that employed ABPM devices capable of recording by both oscillometric and auscultatory methods showed a higher percentage of missed auscultatory compared to oscillometric readings (Keavney et al., 2000; Raikkonen, Matthews, Flory, \& Owens, 1999).

Participant Comfort. Participant comfort is another important consideration when selecting an ambulatory monitor. In the primary care setting, ABPM tends to receive lower patient acceptability ratings than other forms of BP monitoring (Beltman et al., 1996; Little et al., 2002). Common complaints include inconvenience, discomfort, sleep disturbance, noise, pain, skin irritation, and bruising (Beltman et al., 1996). Although ambulatory monitoring in and of itself can be expected to cause participants some degree of discomfort, patient acceptability ratings of individual monitors may differ in terms of comfort and convenience (Westhoff et al., 2005). Monitors that are fitted with D-ring cuffs, for example, facilitate unassisted instrumentation by participants, but such cuffs also may be associated with somewhat greater discomfort on inflation for some individuals. Researchers would benefit from screening potential devices with regard to these issues. Because inconvenience and discomfort may reduce compliance with ABPM, reducing the frequency of these complaints may lead to more complete data collection. Moreover, a more tolerable device may result in less restriction of daily activities and thus provide a better assessment of "typical" daily BP. In the absence of formal published studies comparing monitors of interest on these dimensions, researchers should pilot test selected devices before purchase.

Cost. ABPM research requires a considerable monetary investment. The current price for
ABP monitors ranges from about $\$ 2,600$ to $\$ 3,400$ per unit, depending on the model and choice of accessories.

\section{Additional Considerations}

Cuff Sizing. The consensus recommendation of the American Heart Association (AHA) is that the "ideal" BP cuff should have a bladder width that is at least $40 \%$, and a length that is at least $80 \%$ of the arm circumference (Pickering et al., 2005). A bladder that is too narrow or too short will result in overestimation of BP readings, whereas a bladder that is too wide or too long may cause underestimation of readings. Table 8.1 displays AHA recommended dimensions for adult cuff bladder sizes based on these criteria. It should be emphasized that AHA recommendations are only guidelines. Despite AHA recommendations, bladder dimensions corresponding to small adult, adult, and large adult cuff sizes may differ across manufacturers or within manufacturers over time. When selecting an ABPM device, it is in the best interest of the researcher to choose a manufacturer that produces cuffs with dimensions that correspond to those recommended by the AHA. Given the potential influence of cuff size on BP readings, it is essential that the same bladder size be used if ABPM is conducted during multiple assessment periods. Likewise, bladders of the same size must be used for ABP and clinic BP assessment if both types of data are collected (Staessen, Fagard, Thijs, \& Amery, 1995).

Although the AHA cuff size recommendations are appropriate in most situations, in some instances they may be difficult to apply. For example, the large adult cuff may not be appropriate for obese participants with relatively short upper arms, as the extra width of the large adult cuff may exceed the length of the upper arm (Pickering et al., 2005). A smaller cuff may be more comfortable for these individuals, but its use increases the risk 
Table 8.1 AHA Recommended Cuff Sizes (in $\mathrm{cm}$ )

\begin{tabular}{lcc}
\hline Cuff Size & $\begin{array}{c}\text { Bladder Dimensions, } \\
\text { Width } \times \text { Length }\end{array}$ & Arm Circumference \\
\hline Small adult & $12 \times 22$ & $22-26$ \\
Adult & $16 \times 30$ & $27-34$ \\
Large adult & $16 \times 36$ & $35-44$ \\
Adult thigh & $16 \times 42$ & $45-52$ \\
\hline
\end{tabular}

SOURCE: Adapted from T.G. Pickering et al. (2005).

of overestimating their BPs. In addition to the difficulty associated with fitting cuffs to shorter arms with large circumferences, obese upper arms also may be associated with K-sound detection difficulties, causing problems with auscultatory monitors (Keavney et al., 2000). Thus, researchers should consider the possibility that some individuals may not be able to participate in ABPM study protocols.

Long-Term Availability of Product. Given the substantial investment that is being made in ABPM equipment, it is important for investigators to inquire about the projected longterm availability of the product of interest. Even minor changes in product characteristics might result in incompatibilities between older and newer models. Our laboratory had first-hand experience with model incompatibility when refurbishing our ABPM equipment for the 6-year follow-up of our ongoing study of psychosocial and behavioral influences on ABP. We discovered that the company that supplied our baseline equipment had recently dropped the use of latex bladders. The new bladders, which were nylon, were of slightly different dimensions than the older versions. Accordingly, cuff sizes were altered as well. As a result, we were unable to obtain new bladders that were of the same dimensions as those we used at baseline. In order to maintain consistency across monitoring periods, we were forced to locate new cuffs that could accommodate the old bladders used at baseline. The company was helpful in working with us to resolve this problem, but researchers need to be aware of such difficulties up front, as they typically are not issues of concern to the average customer sales department. When purchasing replacement products for an ongoing cross-sectional or longitudinal study, company representatives should be queried for all potential hardware or software modifications that may have transpired since the original units were purchased (O'Brien et al., 1990).

Available Assessment Schedules. In a later section, we discuss various interval schedules that researchers might employ to collect ABP data. When selecting a monitor, it is important to ensure that the device of interest is capable of administering ABP assessments according to the desired schedule. For example, most units include a design feature that permits an automated "re-try," a reinflation of the cuff following an artifactual reading. For some units, the time interval between readings (e.g., 30 minutes) is calculated relative to the re-try rather than to the original reading. This shift in internal timing can be a problem insofar as the clock time associated with each initial BP initiation "drifts" with each re-try (e.g., BP may be taken at 9:00, 9:30, and 10:00 for one individual, and 9:04 (retry), 9:34, and 10:04 for another). Thus, it becomes difficult to align participants at common time points and to 
synchronize the ABP device with, for example, handheld computers for coordinated program routines. Once again, features such as this one may have little consequence for routine clinical practice, but may be problematic for research. Such features should be tested and inquired about when selecting an ABP monitor.

Option to Turn Off Display. Most, if not all monitors display ABP readings on a small LCD screen. Because participants' knowledge of their BPs might influence subsequent readings, availability of the option to turn off the LCD display is important.

Software. All ABP monitors come with software that performs functions such as plotting ABP profiles, performing selected statistical analyses, and facilitating export of data for more comprehensive research analysis. Researchers might evaluate software products on ease of use, compatibility with platforms in use by analysis workstations, and capacity for data security.

Risk. Risks associated with ambulatory monitoring are few and minor, and typically involve superficial reactions following repeated cuff application, such as skin irritation and bruising (Beltman et al., 1996). Researchers might be advised to consult with individual manufacturers to determine whether any additional risks might be associated with their product.

\section{Training}

Research Staff Training. Research staff should be familiar with principles of conventional BP measurement, such as palpation of the brachial artery, auscultation, and cuff fitting (Perloff et al., 1993). Skill in manual BP measurement techniques is necessary because ambulatory monitors must be calibrated against a manual manometry standard before participants are sent into the field. The protocol for calibration involves connecting the ambulatory device to a mercury sphygmomanometer with a $\mathrm{Y}$ or $\mathrm{T}$ connection. The automatic pressure system and BP detection mechanisms of the ambulatory device are disabled, and thus the device acts simply as a pressure gauge. Pressures within this system are compared to measurements displayed on the mercury column. Using this protocol, BP measurements taken with the ambulatory device must fall within $5 \mathrm{mmHg}$ of a mercury standard (Pickering et al., 2005). In our lab, research associates take two readings of participants' pressures. If either automated measurement fails to meet the $5 \mathrm{mmHg}$ criterion, the monitor is replaced and a second set of calibration procedures is conducted.

Research staff also should understand the procedure for downloading ABP data to a PC, and be fluent in the use of relevant software. We have found it helpful to provide immediate feedback to participants when they returned their monitors to the lab. Being able to produce a readable printout of participants' ambulatory readings facilitates discussions with participants about their BP data, which can be an important motivational factor for study participation.

In addition to being trained in the operation of the ambulatory monitor, staff members should be well versed in the workings of the device, and thus able to troubleshoot problems that might arise for participants while monitoring in the field. Researchers might be advised to place a staff member on call 24 hours a day while participants are monitoring. In our laboratory, oncall staff members receive two to three calls from participants per week. A typical problem reported during these calls is the need for instruction on how to replace the batteries in the monitor. 
Participant Training. Participants should be instructed on proper use and maintenance of the ambulatory monitor. In our laboratory, two research associates conduct training sessions among groups of one to five participants using a standard script and Power Point presentation. Several types of information should be related to participants during training. First of all, participants should be provided with basic information on what to expect while monitoring, such as how frequently BP will be assessed, and how the monitor sounds and feels during cuff inflation. Furthermore, participants should be reminded that the researcher is interested in collecting information on BP during activities that are typical in daily life, and they therefore should try to maintain their usual routines during monitoring. Some participants attempt to adjust their routines to reduce discomfort with the monitor, such as monitoring only during nonwork days. This strategy is obviously not optimal, because BP data obtained during nonwork days may not be generalizable to work days. Moreover, given that full-time employees spend more days during a typical week at work than in nonwork settings, nonwork BPs may not be entirely representative of typical daily pressures.

Also fundamental to participant training is instruction on how to properly remove and replace the compression cuff. Participants should be instructed on where to place the cuff on the arm, and how tightly to wrap the cuff so that it remains stable during monitoring, but does not impair circulation. Use of an auscultatory monitor requires additional training because participants must be instructed on proper positioning of the transducer (microphone). Our research associates use a semipermanent marker to indicate where on the arm the microphone should be placed. Many, if not most, research protocols do not ask participants to perform their own instrumentation. The main benefit of instrumentation in the lab is that trained staff can ensure that cuffs are fitted correctly. However, if monitoring is to take place over several days, having participants return to the lab for instrumentation every morning creates substantial burden.

Another issue that needs to be addressed during training is movement and position of the arm during measurement. Participants should be told to keep their arm immobile while measurements are being made. Participants also should be encouraged to maintain consistent posture throughout the period of measurement, as changes in posture may influence readings (O'Brien et al., 2003). For example, if a participant is standing while SBP is being measured, but seated while DBP is being measured, the resulting pulse pressure will be artificially wide. If participants are undergoing 24-hour monitoring, they can be instructed to place the monitor on the bed or under a pillow (to reduce noise during cuff inflation) when they go to bed at night (O'Brien et al., 2000). Participants should be cautioned against sleeping with the monitored arm on top of the pillow that is covering the monitor, because this vertical displacement of the arm can lead to artificially lower BP readings, and thus create the appearance of exaggerated dipping (Kammila, Campbell, Brant, deJong, \& Culleton, 2002).

Finally, participants should be instructed in the proper care and maintenance of their monitors. Participants should be instructed to keep monitors away from water, to avoid dropping or bumping the monitor, and to avoid pulling or jerking the tubing that connects the monitor to the compression cuff.

We recommend that participants have an opportunity to practice monitoring before data collection begins. We typically follow training with a "shakedown day" and feedback session, which gives participants an opportunity to troubleshoot any difficulties they may have with the monitor. In some instances, the first few readings during an 
ABPM period may be abnormally high, a phenomenon that may result from initial heightened awareness of the monitoring situation (Owens, Lyons, Rodriguez, \& O’Brien, 1998). If such initial readings are included as part of a training period, they can be discarded without loss of actual data.

\section{Artifact}

ABP data generally include a percentage of $\mathrm{BP}$ readings that lie outside of the participant's usual range and likely do not represent genuine BP values. Various editing methods have been designed to identify and eliminate outlying values in the raw ABP data. It is in the best interest of the researcher, however, to identify sources of artifactual readings, and incorporate safeguards into their procedures to minimize the number of outlying values. In other words, concern for outlying values should begin at data collection rather than at data analysis.

Sources of Artifact. Three important sources of artifactual readings are arm movement, cuff or microphone displacement, and position of the arm relative to the heart. Arm movement is a common cause of failed readings (Palatini, 2001), and is especially problematic when an oscillometric device is being used. Motion artifact can be minimized to some extent by instructing participants to abstain from moving the arm to which the cuff is attached while a BP measurement is being taken. By comparison, cuff displacement is a more significant source of error when an auscultatory monitor is being used. Movement of the cuff may result in dislocation of the transducer from the site of the brachial artery, thus hindering the ability to detect K-sounds. Microphone displacement might be avoided by taping the transducer to the participant's arm. In our lab, participants are provided with Lycra sleeves that are designed to be worn under the compression cuff, and contain a small pocket in which to place the transducer.

Handling Artifact. Accurate identification and elimination of outlying values is essential to proper interpretation of ABP data. Rejection of outliers can substantially alter ABP data (Berardi, Chau, Chanudet, Vilar, \& Larroque, 1992; Winnicki, Canali, Mormino, \& Palatini, 1997), even to the point of reducing the ratio of individuals in a given sample whose data meet diagnostic criteria for hypertension (Winnicki et al., 1997). A review of several outlier rejection methods identified three basic approaches: univariate, multivariate, and temporal (Berardi et al., 1992). Univariate approaches reject BP readings if they fall outside of a specified range. The Casadei method, for example, rejects SBP readings that are greater than $240 \mathrm{mmHg}$ or less than $50 \mathrm{mmHg}$ (Casadei et al., 1988). Multivariate approaches examine simultaneous readings of SBP, DBP, and heart rate, and reject values based on some combination of these parameters, such as pulse pressure or the SBP/DBP ratio. More complicated multivariate methods consider the influence of covariates, such as exercise concurrent with the ABP measurement, and incorporate these factors into mathematically complex algorithms (Clark et al., 1987). Temporal approaches are based on the slope of the SBP and DBP versus time curves, and reject BP readings that diverge too far from the associated models.

Comparison across studies of the various outlier rejection methods does not result in the identification of a single set of criteria that is unequivocally superior to others in distinguishing artifactual readings from true ABP values (Berardi et al., 1992). In a single study that compared several editing methods by assessing their effects on a single set of data, Winnicki and colleagues observed that methods may differ with respect to improving the reproducibility of ABP (Winnicki et al., 1997). 
Their results suggested that a modified version of the cutpoints designed by Casadei and colleagues (Casadei et al., 1988) appeared to provide the most reproducible results. The authors acknowledged, however, that reproducibility may not be the most appropriate criterion on which to judge the accuracy of outlier rejection techniques. Judgment might better be based on comparison of edited ABP values with intraarterial BP readings. To date, no published study has compared edited ABP values that have been obtained by various outlier rejection methods with intraarterial measurements. In the absence of empirical evidence to support the superiority of one outlier rejection method over the rest, recent consensus statements (O'Brien et al., 2003; Pickering et al., 2005) have made no recommendations. It has been suggested, however, that if a sufficient number of measurements exist, editing is not necessary to calculate mean ABP values, and only grossly deviant readings should be deleted from the dataset (O’Brien et al., 2003).

\section{Design Issues}

Sample Size. Of fundamental concern when planning an ABPM study is establishing an appropriate sample size. Depending on the research question of interest, the "sample" might be composed of (a) the number of participants who are enrolled in the study, (b) the number of ABP readings per participant, and/or (c) the total number of collected ABP readings (i.e., the number of $\mathrm{ABP}$ readings per participant multiplied by the number of participants in the study). Relevant factors influencing appropriate sample size include generalizability of the ABP data, analytic strategy and desired statistical power, and cost.

Generalizability. The extent to which collected ABP data are generalizable to "typical" daily BPs is determined, in large part, by the number of ABP readings that are collected per subject. Llabre and colleagues applied generalizability theory to BP measurements that were collected in various contexts (Llabre et al., 1988). The results of the analysis suggest that at least 6 replications of SBP and 6 to 10 replications of DBP at work and at home, respectively, may be required to ensure acceptable levels of generalizability to typical pressures in each of those settings (Llabre et al., 1988).

Analytic Strategy and Statistical Power. The preferred method for analyzing ABP data involves the use of complex multilevel models (Schwartz \& Stone, 1998), which are discussed in greater detail in the next section. In reference to these statistical techniques, one frequently cited rule of thumb is that sample sizes should be composed of at least 30 subjects with at least 30 observations per subject (Hox, 2002). This recommendation, of course, is intended only as a general guideline. A more accurate method for determining sample size is to consider the desired power to predict anticipated effects. A discussion of power analysis for multilevel models is beyond the scope of the present chapter. However, detailed information can be obtained elsewhere (Hox, 2002; Raudenbush \& Liu, 2000; Snijders \& Bosker, 1999).

Cost. In addition to considering the factors already discussed, investigators must weigh the cost-in terms of time, money, and participant burden associated with the design, including whether it would be more efficient to include additional persons or additional observations (Hox, 2002).

Frequency and Schedule of Sampling. Studies involving ambulatory data collection might be classified according to whether the initiation of a measurement is contingent on temporal or event-based criteria (Bolger, Davis, \& Rafaeli, 2003). Designs that use temporalbased criteria can be further divided into 
fixed- and variable-schedule protocols. In fixed-schedule designs, assessments are taken either at specific times of the day (e.g., 9:00 a.m., noon, and 4:00 p.m.) or at specific time intervals (e.g., every hour, every 3 hours, daily). The length of the interval between assessments is an important consideration when employing a fixed-schedule design. Intervals that are too long may result in loss of important information, whereas intervals that are too short might increase the signal-tonoise ratio and place unnecessary burden on participants. Bolger and colleagues conclude it is best to err on the side of shorter rather than longer intervals (Bolger et al., 2003). In our research on psychosocial and behavioral correlates of daily $\mathrm{BP}$, we assessed $\mathrm{ABP}$ and concurrent psychological and physical state at 45-minute intervals during waking hours. This interval is slightly longer than those usually employed in ABP studies, which typically range from every 15 to every 30 minutes. We chose 45 minutes rather than a more frequent interval because of the time-consuming nature of the self-report psychosocial and behavioral assessment that our participants completed concurrently with each ABP recording. We avoided a strict hourly interval in order to reduce synchronization with daily activities, such as classes or meetings, which frequently start and stop on the hour.

In variable-schedule designs, assessments of the phenomena of interest are distributed according to a random pattern to which participants are blinded. An advantage associated with variable-schedule designs is that random assessments may reduce potential for biased reporting (Bolger et al., 2003). By way of illustration, consider a study wherein participants are asked to report on the level of stress they are experiencing at the time of each $\mathrm{ABP}$ assessment. If $\mathrm{ABP}$ is assessed at regular intervals, participants might begin to attend specifically to stressful aspects of their experience during times that they anticipate cuff inflation. Assessment at random intervals would reduce the risk of such anticipatory bias. Variable-schedule designs may be associated with greater participant burden, however, because participants may have greater difficulty accommodating to the random distribution of assessments.

In event-based designs, assessments take place only within the context of a clearly defined triggering event. Thus, event-based sampling is appropriate when interest is in assessing the variable of interest during rare or specialized situations that may not be captured with time-based strategies. For example, in their study of social relationships and ABP, Holt-Lunstad and colleagues instructed participants to initiate an ABP reading approximately 5 minutes into every social interaction. Participants also completed a brief paper-andpencil diary report concurrent with each ABP assessment (Holt-Lunstad, Uchino, Smith, Olson-Cerny, \& Nealey-Moore, 2003). From these data, the authors were able to assess whether ABP levels during social interactions differed as a function of (a) interaction partner and (b) affective quality of the interaction (Holt-Lunstad et al., 2003). Bolger and colleagues describe two potential risks associated with event-contingent designs, the first having to do with participant compliance with protocol and the second with investigators' treatment of the data (Bolger et al., 2003). With regard to the first risk, the success of eventcontingent data collection depends largely on the ability of participants to recognize and respond to the trigger event. Thus, insofar as participants are unreliable in either regard, substantial relevant data may be lost. With regard to the second risk, investigators may be tempted to overgeneralize from event-based assessments, which are not strictly representative of participants' general experiences.

Definitions of Daytime and Nighttime. If monitoring is to take place over 24 hours, researchers must determine how best to define periods of daytime wakefulness and 
nighttime rest. As discussed in an earlier section, BP tends to follow a diurnal rhythm wherein pressures are higher during the day and lower at night (the dipping phenomenon), with the primary determinants of this rhythm being posture, activity, and arousal (Pickering, Schwartz, \& Stone, 1996). Inaccurate specification of daytime and nighttime, then, can lead to poor estimation of average day (waking) and night (sleep) pressures. ABPM software packages typically contain a default day-night definition (e.g., day $6: 00$ to $22: 00$; night $22: 00$ to $6: 00$ ). However, evidence suggests that default definitions do not correspond to all participants' actual periods of sleep and wakefulness, and thus "default" day and night pressures may differ markedly from actual day and night values (Eissa, Yetman, Poffenbarger, \& Portman, 1999; Gatzka \& Schmieder, 1995). Thus, researchers might be advised to include measures of participants' actual times of awakening and going to sleep. Several ABPM studies have incorporated self-report diary reports of participants' waking and sleeping times (Gatzka \& Schmieder, 1995; Loimaala et al., 1997; Robles, Cancho, Ruiz-Calero, Angulo, \& Sanchez-Casado, 2003; Shapiro \& Goldstein, 1998). The main advantage of using diary reports rather than default daynight definitions is that diary reports allow for the possibility that participants' true waking and sleeping times differ from (a) the times used to define default day and night periods and (b) other participants' waking and sleeping times. A limitation of this approach is that it creates additional burden for participants.

Another method that might be used to identify participants' true periods of waking and sleeping is asking them to wear an actigraph in addition to the ABP monitor (Eissa, Poffenbarger, \& Portman, 2001; Eissa et al., 1999). Like diary reports, actigraphy data have the advantage of providing individualized information on participants' actual sleep-wake patterns. Moreover, unlike diary methods, actigraphy presents no additional participant burden, save for the wearing of the actigraph. An obvious limitation of using actigraph data to define awake and sleep periods is the cost associated with the purchase of additional equipment.

A novel approach to identifying sleepwake patterns involves analysis of heart rate (HR) data. Pekarski and colleagues developed a mathematical algorithm for identifying nighttime rest as a period of low ambulatory HR values (Pekarski, Mordovin, Triss, Semke, \& Kolodina, 2002). Advantages associated with this method are that examination of HR does not require purchase of additional equipment (discrete HR measures can be obtained from the ABPM device) and it does not place additional burden on participants (Pekarski et al., 2002). On the other hand (for example, in the measurement of BP dipping), such measures may reflect a confound between the independent (activity level) and dependent (cardiovascular assessments) variables of interest.

Monitoring for More Than 24 Hours. The duration of ABPM studies often is limited to a single 24-hour period. Depending on the interests of the researcher, however, there may be some advantage to extending the monitoring period to longer than 24 hours. In one of our studies, for example, we collected ambulatory data (i.e., ABP and concurrent diary data) during two 3-day periods (waking hours only) that were separated by about 4 months. Our intention with this design was to enhance the likelihood that we were capturing a "representative slice" of participants' lives. Holt-Lunstad and colleagues monitored participants during the course of a single 3day period. In this case, a multiple-day monitoring period was selected in order to obtain a broader sampling of participants' social interactions (Holt-Lunstad et al., 2003). Increasing the duration of the monitoring 
period also might be an alternative approach to increasing the power of the study if increasing the frequency of ABP measurements is not feasible (Raudenbush \& Xiao-Feng, 2001).

\section{Handling Metabolic and Behavioral Influences on ABP}

ABP may be influenced substantially by metabolic and behavioral activities that are ongoing at the time a reading is taken. Thus, it may be useful to instruct participants to report on level of activity, posture, or ingestion of food, drink, caffeine, tobacco, or medication concurrent with ABP assessments. A number of ambulatory diaries have been developed for this purpose, and most involve paper-and-pencil recording of metabolic and behavioral influences. An alternative strategy is to use an electronic diary that can be displayed on a handheld computer. Evidence suggests that patient compliance with electronic diaries is superior to that shown with paper diaries (Stone, Shiffman, Schwartz, Broderick, \& Hufford, 2002). In one of our recent studies, participants provided, on average, 105 complete observations with valid electronic diary and ABP readings, representing $75 \%$ of all possible observations over two 3-day monitoring periods, which suggests that such systems are feasible and can be associated with a reasonable level of compliance (Kamarck et al., 2007). Accelerometry-based physical activity monitors also may be used for indexing physical activity correlates of ABP (Kario, Schwartz, et al., 2001).

\section{ANALYTIC ISSUES}

To fully appreciate the wealth of information that ABPM data have to offer, researchers must acknowledge the complex, multilevel structure of the data and employ analytic techniques that can accommodate that structure.

\section{Structure of ABP Data and Questions That Can Be Asked of It}

ABPM is one example of ecological momentary assessment (EMA) (Stone \& Shiffman, 1994), which involves collecting repeated measurements of behavioral, psychological, or physiological phenomena as they occur in the natural setting. An important feature of EMA research is two-stage sampling. For example, in an ABP study, the first stage occurs when participants are sampled from the larger population of individuals; in the second stage, ABP measurements are sampled from the larger population of participants' daily pressures. In this way, data from ABPM research comprise a hierarchical system (Hox, 2002) wherein ABP measurements are "nested" within participants.

The multilevel structure of ABP data suggests at least two sources of random variation: between-person and within-person. Thus, ambulatory monitoring allows researchers to examine the effects of between- and withinperson differences on daily BP, as well as potential cross-level interactions. In their excellent discussion of analytic strategies for use in EMA research, Schwartz and Stone describe three types of questions that multilevel data might address: (1) Do participant characteristics (between-person factors) predict differences in mean levels of an outcome? (2) do contextual features (within-person factors) predict momentary fluctuations among individual assessments? and (3) do participant characteristics predict differences in within-person associations? (Schwartz \& Stone, 1998). Specifically with regard to ABP research, one might investigate whether a given psychological trait, such as hostility, predicts between-person differences in average ABP (Raikkonen et al., 1999). One also might examine whether momentary reports of social conflict at the time of ABP measurement predict within-person changes 
in momentary ABP levels, and whether that within-person association depends on betweenperson differences in hostility (Brondolo et al., 2003).

A number of statistical techniques that employ ordinary least squares (OLS) estimation have been used to analyze multilevel data like those obtained from ABPM. These include ordinary analysis of variance (ANOVA) or covariance (ANCOVA), nested ANOVA and ANCOVA, and pooled within-person regression analysis. Schwartz and Stone provide a detailed description of these techniques, and the extent to which each is more or less appropriate for analysis of EMA data (Schwartz \& Stone, 1998). In brief, the authors concluded that the majority of standard statistical methods are not appropriate for use in EMA research because multilevel data frequently exhibit one or more of the following features: nonindependence, autocorrelation, unbalanced design, and heteroscedasticity.

Nonindependence. Because ABP readings are nested within participants, individual ABP observations are not completely independent. Specifically, average correlations between ABP measurements that are taken from a single individual will tend to be higher than correlations between ABP measurements taken from different individuals. Standard OLS statistics rely on the assumption of independence of observations. Violation of this assumption results in standard errors that are too small, and thus a higher probability of Type 1 error.

Autocorrelation. Because each participant's ABP measurements are collected sequentially, the residuals will tend to show a certain degree of autocorrelation. In other words, for a given participant, ABP readings taken within close temporal proximity will tend to be more similar than ABP readings that are separated by a large temporal distance.
Failure to control for autocorrelation tends to result in overestimation of between-person variability, underestimation of within-person variability, and increased risk of Type 1 error in the analysis of within-person effects (Schwartz \& Stone, 1998).

Unbalanced Design and Heteroscedasticity. Balanced designs are rare among ABPM studies because data are frequently missing, thus leading to unequal numbers of observations between participants. As within-person variability is determined, in part, by the number of observations, unequal numbers of observations across participants will lead to unequal error variances, or heteroscedasticity. Heteroscedasticity in the data reduces the efficiency of estimates of model parameters, resulting in tests of significance that can elevate the possibility of either Type 1 or Type 2 error, depending on the pattern of heterogeneity.

\section{Multilevel Modeling}

Multilevel models that use maximum likelihood (ML) estimating techniques have been recommended as an alternative to conventional OLS statistics for analyzing EMA data (Schwartz \& Stone, 1998). One of the main advantages of multilevel models is that they use all available data, rather than excluding cases with missing observations. In other words, they can accommodate unbalanced designs. This feature of multilevel models is of particular relevance to ABP data, as it is common for a significant proportion of participants to be missing at least one observation. Another advantage of multilevel models is that they allow for the specification of error variance, and thus permit accurate and efficient effect estimation in the presence of heteroscedasticity, which is common to ABP data. An additional advantage of multilevel models is that they permit the modeling of 
autocorrelation. More thorough discussions of the theory behind multilevel models and associated analytic techniques is provided elsewhere (Bryk \& Raudenbush, 1992; Hox, 2002; Singer \& Willett, 2003; Snijders \& Bosker, 1999).

\section{Available Software}

Several statistical packages capable of performing multilevel analyses with maximum likelihood estimation have become available during the past decade, and a number of these have been reviewed (Kreft, deLeeuw, \& Vander Leeden, 1994; Zhou, Perkins, \& Hui, 1999). Currently, HLM (Bryk, Raudenbush, \& Congdon, 1996) and MLwinN (Rasbash, Steele, \& Browne, 2003) are the two major specialized programs. Less well known specialized programs include MIXREG (Hedeker \& Gibbons, 1996b) and MIXOR (Hedeker \& Gibbons, 1996a), for continuous and dichotomous or ordinal data, respectively. Also available are multilevel modules included in major statistical packages, such as the SAS multilevel procedure, PROC MIXED (Littell, Milliken, Stroup, \& Wolfinger, 1996; SAS Institute, 1992), and the SPSS multilevel procedure, MIXED (SPSS for Windows, Rel. 11.5 2001. Chicago: SPSS Inc). One advantage of the SAS and SPSS procedures is that a single statistical package can be used for management and analysis of multilevel data. Singer has written an excellent tutorial for using SAS PROC MIXED for fitting multilevel models (Singer, 1998). A similar tutorial for SPSS MIXED is provided by Peugh and Enders, who duplicate Singer's analyses using the SPSS procedure (Peugh \& Enders, 2005).

\section{REPORTING FINDINGS FROM ABPM RESEARCH}

Given the methodological rigor of ABPM research and the complexity of $\mathrm{ABP}$ data, special care must be taken to include appropriate detail when presenting the findings of an ABPM study. Stone and Shiffman have written an excellent paper that outlines several features of EMA research, in general, that would be helpful to discuss in reports of EMA study findings (Stone \& Shiffman, 2002). These features include (1) the rationale for choosing an EMA design, (2) the procedure for collecting EMA data, (3) details of the monitoring apparatus, (4) participant compliance with the sample procedure, (5) participant training, (6) data management procedures, and (7) the data analytic approach (Stone \& Shiffman, 2002).

\section{CONCLUSION}

The ability to assess BP in the field as individuals go about their daily routines provides an opportunity for behavioral medicine researchers to identify psychosocial correlates of fluctuations in cardiovascular activity. Given the demonstrated prognostic utility of ABPM, the ability to identify psychosocial and behavioral correlates of daily pressures ultimately may lead to a better understanding of the role played by these factors in the emergence of clinical CVD.

In exchange for the potential benefits afforded by ABPM, researchers must be willing to invest considerable time and resources in preparation for and in the actual conduct of an ABPM study. Researchers must be educated in which factors to consider when selecting an ambulatory monitor and be prepared to make a substantial initial monetary investment in equipment. Research staff must be trained in the operation and maintenance of ABPM equipment, and be able to answer questions and troubleshoot any problems that might arise while research participants are monitoring. Research participants, as well, must be trained in the proper care and use of their monitors. The design of an 
ABPM study must be informed by issues pertaining to definitions of daytime and nighttime, whether monitoring will take place for more than 24-hours, and how best to measure the effects of behavioral factors (such as posture and ingestion of food or drink) on
ABP. Finally, researchers must be educated in how to interpret, edit, and analyze the ABP data they have collected. It is our hope that the information provided in this chapter will aid interested investigators in furthering research in this area.

\section{SUGGESTED READINGS}

Kamarck, T. W., Schwartz, J., Shiffman, S., Muldoon, M. F., Sutton-Tyrrell, K., \& Janicki, D. L. (2005). Psychosocial stress and cardiovascular risk: What is the role of daily experience? Journal of Personality, 73, 1749-1774.

O’Brien, E., Asmar, R., Beilin, L., Imai, Y., Mallion, J.-M., Mancia, G., et al. (2003). European Society of Hypertension recommendations for conventional, ambulatory and home blood pressure measurement. Journal of Hypertension, 21(5), 821-848.

Pickering, T. G., Hall, J. E., Appel, L. J., Falkner, B. E., Graves, J., Hill, M. N., et al. (2005). Recommendations for blood pressure measurement in humans and experimental animals part I: Blood pressure measurement in humans. Hypertension, 45, 142-161.

Pickering, T. G., \& Kario, K. (2001). Nocturnal non-dipping: What does it augur? Current Opinion in Nephrology \& Hypertension, 10(5), 611-616.

Schwartz, J. E., \& Stone, A. A. (1998). Strategies for analyzing ecological momentary assessment data. Health Psychology, 17(1), 6-16.

1. Carotid IMT has been shown to be associated with autopsy measures of atherosclerosis (Pignoli, Tremoli, Poli, Oreste, \& Raoletti, 1986; Wong, Edelstein, Wollman, \& Bond, 1993), and to correlate with risk for heart attack and stroke (Aronow \& Schoenfeld, 1992; Hodis, Mack, \& LaBree, 1998; O’Leary, Polak, \& Kronmal, 1999; Salonen \& Salonen, 1991; Sutton-Tyrrell, Alcorn, Herzog, Kelsey, \& Kuller, 1995).

\section{REFERENCES}

Amar, J., Vernier, I., Rossignol, E., Bongard, V., Arnaud, C., Conte, J. J., et al. (2000). Nocturnal blood pressure and 24-hour pulse pressure are potent indicators of mortality in hemodialysis patients. Kidney International, 57(6), 2485-2491.

Aronow, W. S., \& Schoenfeld, M. R. (1992). Forty-five-month follow-up of extracranial carotid arterial disease for new coronary events in elderly patients. Coronary Artery Disease, 3, 249-251.

Association for the Advancement of Medical Instrumentation. (1993). American national standard. Electronic or automated sphygmomanometers. ANSI/AAMI SP 10-1992. Arlington, VA: AAMI. 
Beltman, F. W., Heesen, W. F., Smit, A. J., May, J. F., Lie, K. I., \& Meyboom-de Jong, B. (1996). Acceptance and side effects of ambulatory blood pressure monitoring: Evaluation of a new technology. Journal of Human Hypertension, 10(suppl 3), S39-S42.

Berardi, L., Chau, N. P., Chanudet, X., Vilar, J., \& Larroque, P. (1992). Ambulatory blood pressure monitoring: A critical review of the current methods to handle outliers. Journal of Hypertension, 10, 1243-1248.

Bianchi, S., Bigazzi, R., Baldari, G., Sgherri, G., \& Campese, V. M. (1994). Diurnal variations of blood pressure and microalbuminuria in essential hypertension. American Journal of Hypertension, 7(1), 23-29.

Bobrie, G., Chatellier, G., Genes, N., Clerson, P., Vaur, L., Vaisse, B., et al. (2004). Cardiovascular prognosis of "masked hypertension" detected by blood pressure self-measurement in elderly treated hypertensive patients. JAMA, 291, 1342-1349.

Bolger, N., Davis, A., \& Rafaeli, E. (2003). Diary methods: Capturing life as it is lived. Annual Review of Psychology, 54, 579-616.

Brondolo, E., Rieppi, R., Erickson, S. A., Bagiella, E., Shapiro, P. A., McKinley, P., et al. (2003). Hostility, interpersonal interactions, and ambulatory blood pressure. Psychosomatic Medicine, 65, 1003-1011.

Bryk, A. S., \& Raudenbush, S. W. (1992). Hierarchical linear models. London: Sage.

Bryk, A. S., Raudenbush, S. W., \& Congdon, R. J. (1996). HLM: Hierarchical linear and nonlinear modeling with the HLM/2L and HLM/3L programs. Chicago: Scientific Software International.

Casadei, B., Parati, G., Pomidossi, G., Groppelli, A., Trazzi, S., Di Rienzo, M., et al. (1988). 24-hour blood pressure monitoring: Evaluation of Spacelabs 5300 monitor by comparison with intra-arterial blood pressure recording in ambulant subjects. Journal of Hypertension, 6(10), 797-803.

Clark, L. A., Denby, L., Pregibon, D., Harshfield, G. A., Pickering, T. G., Blank, S., et al. (1987). A data-based method for bivariate outlier detection: Application to automatic blood pressure recording devices. Psychophysiology, 24(1), 119-125.

Clement, D. L., De Buyzere, M. L., De Bacquer, D. A., de Leeuw, P. W., Duprez, D. A., Fagard, R. H., et al. (2003). Prognostic value of ambulatory blood-pressure recordings in patients with treated hypertension. New England Journal of Medicine, 348(24), 2407-2415.

CMS. (2001). Centers for Medicare \& Medicaid Services. Medicare coverage policy一decisions. ABPM Monitoring (\#CAG-00067N). Accessed at http://www .hcfa.gov/coverage/8b3-ff.htm

dabl Educational Trust. Accessed November 15, 2005 at http://www.dabl educational.org/sphygmomanometers.html

Devereux, R. B., Pickering, T. G., Harshfield, G. A., Kleinert, H. D., Denby, L., Clark, L., et al. (1983). Left ventricular hypertrophy in patients with hypertension: Importance of blood pressure response to regularly recurring stress. Circulation, 68(3), 470-476.

Eissa, M. A. H., Poffenbarger, T., \& Portman, R. J. (2001). Comparison of the actigraph versus patients' diary information in defining circadian time periods for analyzing ambulatory blood pressure monitoring data. Blood Pressure Monitoring, 6(1), 21-25.

Eissa, M. A. H., Yetman, R. J., Poffenbarger, T., \& Portman, R. J. (1999). Comparison of arbitrary definitions of circadian time periods with those determined by 
wrist actigraphy in analysis of ABPM data. Journal of Human Hypertension, 13, 759-763.

Fagard, R., Bielen, E., \& Amery, A. (1992). Automated versus observer blood pressure as determinants of left ventricular structure. European Heart Journal, 13, 1373-1379.

Fagard, R. H., Staessen, J. A., \& Thijs, L. (1997). Prediction of cardiac structure and function by repeated clinic and ambulatory blood pressure. Hypertension, $29,22-29$.

Fagard, R., Staessen, J. A., Thijs, L., \& Amery, A. (1995). Multiple standardized clinic blood pressures may predict left ventricular mass as well as ambulatory monitoring: A metaanalysis of comparative studies. American Journal of Hypertension, 8, 533-540.

Fahrenberg, J., \& Myrtek, M. (Eds.). (2001). Progress in ambulatory assessment: Computer-assisted psychological and psychophysiological methods in monitoring and field studies. Ashland, $\mathrm{OH}$ : Hogrefe \& Huber.

Gatzka, C. D., \& Schmieder, R. E. (1995). Improved classification of dippers by individualized analysis of ambulatory blood pressure profiles. American Journal of Hypertension, 8(suppl 7), 666S-671S.

Hall, M., Vasko, R., Buysse, D., Ombao, H., Chen, Q., Cashmere, J. D., et al. (2004). Acute stress affects heart rate variability during sleep. Psychosomatic Medicine, 66, 56-62.

Hansen, T., Jeppesen, J., Rasmussen, S., Ibsen, H., \& Torp-Pedersen, C. (2005). Ambulatory blood pressure and mortality: A population-based study. Hypertension, 45(4), 499-504.

Hedeker, D., \& Gibbons, R. D. (1996a). MIXOR: A computer program for mixedeffects ordinal probit and logistic regression analysis. Computer Methods and Programs in Biomedicine, 49, 157-176.

Hedeker, D., \& Gibbons, R. D. (1996b). MIXREG: A computer program for mixed-effects regression analysis with autocorrelated errors. Computer Methods and Programs in Biomedicine, 49, 229-252.

Henschel, A., de la Vega, F., \& Taylor, H. L. (1954). Simultaneous direct and indirect blood pressure measurements in man at rest and work. Journal of Applied Physiology, 6, 506-508.

Hodis, H. N., Mack, W. J., \& LaBree, L. (1998). The role of carotid artery intimamedia thickness in predicting clinical coronary events. Annals of Internal Medicine, 128, 262-269.

Holt-Lunstad, J., Uchino, B. N., Smith, T. W., Olson-Cerny, C., \& Nealey-Moore, J. B. (2003). Social relationships and ambulatory blood pressure: Structural and qualitative predictors of cardiovascular function during everyday social interactions. Health Psychology, 22(4), 388-397.

Hox, J. (2002). Multilevel Analysis: Techniques and applications. Mahwah, NJ: Lawrence Erlbaum.

Imai, Y., Abe, K., Sasaki, S., Minami, N., Munakata, M., Sakuma, H., et al. (1989). Clinical evaluation of semiautomatic and automatic devices for home blood pressure measurement: Comparison between cuff-oscillometric and microphone methods. Journal of Hypertension, 7, 983-990.

Ituarte, P. H. G., Kamarck, T. W., Thompson, H. W., \& Bacanu, S. (1999). Psychosocial mediators of racial differences in nighttime blood pressure dipping among normotensive adults. Health Psychology, 18, 393-402. 
Joint National Committee. (1997). The 6th report of the Joint National Committee on Detection, Evaluation, and Treatment of High Blood Pressure. Archives of Internal Medicine, 157, 2413-2446.

Kamarck, T. W., Muldoon, M. F., Shiffman, S., Sutton-Tyrrell, K., Gwaltney, C., \& Janicki, D. L. (2004). Experiences of demand and control in daily life as correlates of subclinical carotid atherosclerosis in a healthy older sample. Health Psychology, 23(1), 24-32.

Kamarck, T. W., Polk, D. E., Sutton-Tyrrell, K., \& Muldoon, M. F. (2002). The incremental value of ambulatory blood pressure persists after controlling for methodological confounds: Associations with carotid atherosclerosis in a healthy sample. Journal of Hypertension, 20(8), 1535-1541.

Kamarck, T. W., Schwartz, J., Shiffman, S., Muldoon, M. F., Sutton-Tyrrell, K., \& Janicki, D. L. (2005). Psychosocial stress and cardiovascular risk: What is the role of daily experience? Journal of Personality, 73, 1749-1774.

Kamarck, T. W., Shiffman, S. S., Muldoon, M. F., Sutton-Tyrrell, K., Gwaltney, C. J., Janicki, D. L., et al. (2007). Ecological momentary assessment as a resource for social epidemiology. In A. Stone, S. Shiffman, A. Atienza, \& R. Nebeling (Eds.), The science of real-time data capture: Self-report in health research. Oxford, UK: Oxford University Press.

Kammila, S., Campbell, N. R., Brant, R., deJong, R., \& Culleton, B. (2002). Systematic error in the determination of nocturnal blood pressure dipping status by ambulatory blood pressure monitoring. Blood Pressure Monitoring, 7(2), 131-134.

Kario, K., Pickering, T. G., Matsuo, T., Hoshide, S., Schwartz, J. E., \& Shimada, K. (2001). Stroke prognosis and abnormal nocturnal blood pressure falls in older hypertensives. Hypertension, 38(4), 852-857.

Kario, K., Schwartz, J. E., Davidson, K. W., \& Pickering, T. G. (2001). Gender differences in associations of diurnal blood pressure variation, awake physical activity, and sleep quality with negative affect: The work site blood pressure study. Hypertension, 38(5), 997-1002.

Keavney, B., Bird, R., Caiazza, A., Casadei, B., \& Conway, J. (2000). Measurement of blood pressure using the auscultatory and oscillometric methods in the same cuff deflation: Validation and field trial of the A\&D TM2421 monitor. Journal of Human Hypertension, 14, 573-579.

Khattar, R. S., Senior, R., \& Lahiri, A. (1998). Cardiovascular outcome in whitecoat versus sustained mild hypertension: A 10-year follow-up study. Circulation, 98, 1892-1897.

Kreft, I. G. G., deLeeuw, J., \& Vander Leeden, R. (1994). Review of five multilevel analysis programs: BMDP-5V, GENMOD, HLM, ML3, VARCL. American Statistician, 48(324-335).

Littell, R. C., Milliken, G. A., Stroup, W. W., \& Wolfinger, R. D. (1996). SAS system for mixed models. Cary, NC: SAS Institute.

Little, P., Barnett, J., Barnsley, L., Marjoram, J., Fitzgerald-Barron, A., \& Mant, D. (2002). Comparison of acceptability of and preferences for different methods of measuring blood pressure in primary care. British Medical Journal, $325(7358), 258-259$.

Llabre, M. M., Ironson, G. H., Spitzer, S. B., Gellman, M. D., Weidler, D. J., \& Schneiderman, N. (1988). How many blood pressure measurements are enough? An application of generalizability theory to the study of blood pressure reliability. Psychophysiology, 25(1), 97-106. 
Loimaala, A., Turjanmaa, V., Vuori, I., Oja, P., Pasanen, M., \& Uusitalo, A. (1997). Variation of ambulatory blood pressure in healthy middle-aged men. Journal of Human Hypertension, 11(4), 227-231.

Mancia, G., Di Rienzo, M., \& Parati, G. (1993). Ambulatory blood pressure monitoring: Use in hypertension research and clinical practice. Hypertension, 21, 500-524.

Mancia, G., \& Parati, G. (2000). Ambulatory blood pressure monitoring and organ damage. Hypertension, 36, 894-900.

O'Brien, E., Asmar, R., Beilin, L., Imai, Y., Mallion, J.-M., Mancia, G., et al. (2003). European Society of Hypertension recommendations for conventional, ambulatory and home blood pressure measurement. Journal of Hypertension, 21(5), 821-848.

O’Brien, E., Coats, A., Owens, P., Petrie, J., Padfield, P. L., Littler, W. A., et al. (2000). Use and interpretation of ambulatory blood pressure monitoring: Recommendations of the British Hypertension Society. British Medical Journal, 320, 1128-1134.

O’Brien, E., Petrie, J., Littler, W., de Swiet, M., Padfield, P. L., O’Malley, K., et al. (1990). The British Hypertension Society protocol for the evaluation of automated and semi-automated blood pressure measuring devices with special reference to ambulatory systems. Journal of Hypertension, 8 , 607-619.

O'Brien, E., Petrie, J., Littler, W. A., de Swiet, M., Padfield, P. L., Altman, D., et al. (1993). The British Hypertension Society protocol for the evaluation of blood pressure measuring devices. Journal of Hypertension, 11(suppl 2), S43-S63.

O’Brien, E., Sheridan, J., \& O’Malley, K. (1988). Dippers and non-dippers. Lancet, 2, 397.

O’Brien, E., Waeber, B., Parati, G., Staessen, J. A., \& Myers, M. G. (2001). Blood pressure measuring devices: Recommendations of the European Society of Hypertension. British Medical Journal, 322, 531-536.

Ohkubo, T., Hozawa, A., Nagai, K., Kikuya, M., Tsuji, I., Ito, S., et al. (2000). Prediction of stroke by ambulatory blood pressure monitoring versus screening blood pressure measurement in a general population: The Ohasama Study. Journal of Hypertension, 18, 847-854.

Ohkubo, T., Imai, Y., Tsuji, I., Nagai, K., Watanabe, N., Minami, N., et al. (1997). Relation between nocturnal decline in blood pressure and mortality. The Ohasama Study. American Journal of Hypertension, 10(11), 1201-1207.

O’Leary, D., Polak, J. F., \& Kronmal, R. A. (1999). Carotid-artery intima and media thickness as a risk factor for myocardial infarction and stroke in older adults. New England Journal of Medicine, 340, 14-22.

Owens, P. E., Lyons, S. P., Rodriguez, S. A., \& O'Brien, E. T. (1998). Is elevation of clinic blood pressure in patients with white coat hypertension who have normal ambulatory blood pressure associated with target organ changes? Journal of Human Hypertension, 12, 743-748.

Palatini, P. (2001). Reliability of ambulatory blood pressure monitoring. Blood Pressure Monitoring, 6, 291-295.

Pannarale, G., Bebb, G., Clark, S., Sullivan, A., Foster, C., \& Coats, A. J. (1993). Bias and variability in blood pressure measurement with ambulatory recorders. Hypertension, 22, 591-598.

Pekarski, S. E., Mordovin, V. F., Triss, S. V., Semke, G. V., \& Kolodina, M. V. (2002). Improved automatic analysis of ambulatory blood pressure data based 
on precise detection of individual night-time from diurnal profile of heart rate. Blood Pressure Monitoring, 7, 117-121.

Perloff, D., Grim, C., Flack, J., Frohlich, E. D., Hill, M. N., McDonald, M., et al. (1993). Human blood pressure determination by sphygmomanometry. Circulation, 88, 2460-2470.

Perloff, D., Sokolow, M., \& Cowan, R. (1991). The prognostic value of ambulatory blood pressure monitoring in treated hypertensive patients. Journal of Hypertension, 9(1), S33-S39.

Peugh, J. L., \& Enders, C. K. (2005). Using the SPSS MIXED procedure to fit crosssectional and longitudinal multilevel models. Educational and Psychological Measurement, 65(5), 717-741.

Pickering, T. G. (1996). Recommendations for the use of home (self. and ambulatory blood pressure monitoring. American Journal of Hypertension, 9, 1-11.

Pickering, T. G., Hall, J. E., Appel, L. J., Falkner, B. E., Graves, J., Hill, M. N., et al. (2005). Recommendations for blood pressure measurement in humans and experimental animals part I: Blood pressure measurement in humans. Hypertension, 45, 142-161.

Pickering, T. G., \& Kario, K. (2001). Nocturnal non-dipping: what does it augur? Current Opinion in Nephrology \& Hypertension, 10(5), 611-616.

Pickering, T. G., Schwartz, J. E., \& Stone, A. (1996). Behavioral influences on diurnal blood pressure rhythms. Annals of the New York Academy of Sciences, 783, 132-140.

Pignoli, P., Tremoli, E., Poli, A., Oreste, P., \& Raoletti, R. (1986). Intimal plus medial thickness of the arterial wall: A direct measurement with ultrasound imaging. Circulation, 74, 1399-1406.

Raikkonen, K., Matthews, K. A., Flory, J. D., \& Owens, J. F. (1999). Effects of hostility on ambulatory blood pressure and mood during daily living in healthy adults. Health Psychology, 18(1), 44-53.

Rasbash, J., Steele, F., \& Browne, W. (2003). A user's guide to MLwiN version 2.0. London: Institute of Education, University of London.

Raudenbush, S. W., \& Liu, X. (2000). Statistical power and optimal design for multisite randomized trials. Psychological Methods, 5(2), 199-213.

Raudenbush, S. W., \& Xiao-Feng, L. (2001). Effects of study duration, frequency of observation, and sample size on power in studies of group differences in polynomial change. Psychological Methods, 6(4), 387-401.

Redon, J., Campos, C., Narciso, M. L., Rodicio, J. L., Pascual, J. M., \& Ruilope, L. M. (1998). Prognostic value of ambulatory blood pressure monitoring in refractory hypertension: A prospective study. Hypertension, 31(2), 712-718.

Robles, N. R., Cancho, B., Ruiz-Calero, R., Angulo, E., \& Sanchez-Casado, E. (2003). Nighttime blood pressure fall in renal disease patients. Renal Failure, 25(5), 829-837.

Salonen, R., \& Salonen, J. T. (1991). Determinants of carotid intima-media thickness: A population-based ultrasonography study in Eastern Finnish men. Journal of Internal Medicine, 229, 225-231.

SAS Institute. (1992). SAS/STAT software: Changes and enhancements (SAS Technical Report P-229) (Release 6.07) [Computer software manual]). Cary, NC: Author.

Schwartz, J. E., \& Stone, A. A. (1998). Strategies for analyzing ecological momentary assessment data. Health Psychology, 17(1), 6-16. 
Sega, R., Facchetti, R., Bombelli, M., Cesana, G., Corrao, G., Grassi, G., et al. (2005). Prognostic value of ambulatory and home blood pressures compared with office blood pressure in the general population: Follow-up results from the Pressioni Arteriose Monitorate e Loro Associazioni (PAMELA) study. Circulation, 111(14), 1777-1783.

Shapiro, D., \& Goldstein, I. B. (1998). Wrist actigraph measures of physical activity level and ambulatory blood pressure in healthy elderly persons. Psychophysiology, 35(3), 305-312.

Shiffman, S., \& Stone, A. A. (1998). Introduction to the Special Section: Ecological momentary assessment in health psychology. Health Psychology, 17(1), 3-5.

Singer, J. D. (1998). Using SAS PROC MIXED to fit multilevel models, hierarchical models, and individual growth models. The Journal of Educational and Behavioral Statistics, 24, 323-355.

Singer, J. D., \& Willett, J. B. (2003). Applied longitudinal data analysis: Modeling change and event occurrence. New York: Oxford University Press.

Snijders, T. A. B., \& Bosker, R. (1999). Multilevel analysis: An introduction to basic and advanced multilevel modeling. London: Sage Publications.

Sokolow, M., Werdegar, D., Kain, H. K., \& Hinman, A. T. (1966). Relationship between level of blood pressure measured casually and by portable recorders and severity of complications in essential hypertension. Circulation, 34(2), 279-298.

Staessen, J. A., Fagard, R., Thijs, L., \& Amery, A. (1995). A consensus view on the technique of ambulatory blood pressure monitoring. Hypertension, 26, 912-918.

Staessen, J. A., O’Brien, E. T., Thijs, L., \& Fagard, R. H. (2000). Modern approaches to blood pressure measurement. Occupational and Environmental Medicine, 57, 510-520.

Staessen, J. A., Thijs, L., Fagard, R., O’Brien, E. T., Clement, D., de Leeuw, P. W., et al. (1999). Predicting cardiovascular risk using conventional vs ambulatory blood pressure in older patients with systolic hypertension. JAMA, 282, 539-546.

Stone, A. A., \& Shiffman, S. (1994). Ecological momentary assessment (EMA) in behavioral medicine. Annals of Behavioral Medicine, 16(3), 199-202.

Stone, A. A., \& Shiffman, S. (2002). Capturing momentary, self-report data: A proposal for reporting guidelines. Annals of Behavioral Medicine, 24(3), 236-243.

Stone, A. A., Shiffman, S., Schwartz, J. E., Broderick, J. E., \& Hufford, M. R. (2002). Patient non-compliance with paper diaries. British Medical Journal, 324, 1193-1194.

Sturrock, N. D., George, E., Pound, N., Stevenson, J., Peck, G. M., \& Sowter, H. (2000). Non-dipping circadian blood pressure and renal impairment are associated with increased mortality in diabetes mellitus. Diabetic Medicine, 17(5), 360-364.

Sutton-Tyrrell, K., Alcorn, H. G., Herzog, H., Kelsey, S. F., \& Kuller, L. H. (1995). Morbidity, mortality, and antihypertensive treatment effects by extent of atherosclerosis in older adults with isolated systolic hypertension. Stroke, 26, 1319-1324.

Verdecchia, P., Porcellati, C., Schillaci, G., Borgioni, C., Ciucci, A., Battistelli, M., et al. (1994). Ambulatory blood pressure. An independent predictor of prognosis in essential hypertension. Hypertension, 24(6), 793-801. 